\title{
Draft Genome Resource for Macrophomina phaseolina Associated With Charcoal Rot in Sorghum
}

\author{
Neeraj Purushotham, ${ }^{1}$ Ashley Jones, ${ }^{2}$ Barsha Poudel, ${ }^{1}$ Jamila Nasim, ${ }^{2}$ Dante Adorada, ${ }^{1}$ Adam \\ Sparks, ${ }^{1}$ Benjamin Schwessinger, ${ }^{2}$ and Niloofar Vaghefi ${ }^{1, \dagger}$ \\ ${ }^{1}$ The Centre for Crop Health, University of Southern Queensland, Toowoomba, Queensland, Australia \\ ${ }^{2}$ Research School of Biology, Australian National University, Canberra, Australia
}

\begin{abstract}
Macrophomina phaseolina is a soil-borne phytopathogenic fungus that causes charcoal rot in several plant species, including sorghum. We constructed a draft genome of $M$. phaseolina isolate BRIP 70780a from sorghum, using long-read native DNA from MinION sequencing, which was error-corrected using short-read Illumina MiSeq reads. The draft genome, consisting of 22 contigs with an $N_{50}$ of $4,257,441 \mathrm{bp}, 99.3 \%$ complete benchmarking universal single-copy orthologs, and 14,471 genes, is a valuable resource to aid future studies in population genomics and molecular diagnostic marker development for rapid detection of the pathogen.
\end{abstract}

\section{Genome Announcement}

Macrophomina phaseolina is a polyphagous, soilborne, necrotrophic, phytopathogenic fungus that causes diseases such as seedling blight, charcoal rot, and stem and root rot in more than 500 cultivated plant species (Islam et al. 2012). Charcoal rot is a disease with a high economic impact especially in sorghum, soybean, and maize (Bandara et al. 2018; Islam et al. 2012). In sorghum, the onset of charcoal rot usually occurs after flowering and at the grain filling stage, when the plants are subjected to drought stress. The major symptoms of charcoal rot are degradation of the pith at the crown region and the presence of black microsclerotia, which causes the rotten tissue to appear sooty (Bandara et al. 2018; Sarr et al. 2014). The only published genomes of M. phaseolina include strains MS6, 11-12, and Al-1, isolated from jute, strawberry, and alfalfa, respectively, with genome sizes ranging from 48.9 to $51.3 \mathrm{Mb}$ (Burkhardt et al. 2019; Hossen et al. 2019; Islam et al. 2012), indicating genome plasticity and high evolutionary potential of this pathogen.

The M. phaseolina isolate BRIP 70780a (=MP2) was isolated from charcoal rot-infected sorghum stubble and was cultured in potato dextrose broth for 5 to 7 days at $22^{\circ} \mathrm{C}$ on an orbital shaker (Ratek orbital shaker EOM5) at $120 \mathrm{rpm}$, and mycelia were harvested by filtration. To establish the identity of the isolate as $M$. phaseolina, genomic DNA was extracted using DNeasy plant mini kit (Qiagen) according to manufacturer instructions and the internal transcribed spacers and the intervening 5.8S region of the nuclear ribosomal DNA (de Hoog and Gerrits van den Ende 1998; White et al. 1990), translation elongation factor 1- $\alpha$ (Carbone and Kohn 1999), $\beta$-tubulin (Crous et al. 2004; Woudenberg et al. 2009), and calmodulin (Carbone and Kohn 1999) regions were sequenced (GenBank accession numbers MN636633 and MN640771 to MN640773). A phylogenetic analysis performed in RAxML v.8

\footnotetext{
${ }^{\dagger}$ Corresponding author: N. Vaghefi; niloofar.vaghefi@usq.edu.au
}

The author(s) declare no conflict of interest.

Accepted for publication 23 February 2020.
Funding

This research was funded and supported by the Grains Research and Development Corporation (projects GRDC 9176684, DAQ00194, and DAQ00186), Australia.

\section{Keywords}

Macrophomina phaseolina, sorghum, charcoal rot, MinlON, Oxford Nanopore 
Table 1. Genome statistics for Macrophomina phaseolina isolate BRIP 70780a, constructed using a combination of short-read Illumina and longread MinION sequence data

\begin{tabular}{lccccccc} 
Isolate & Location & Year isolated & Number of contigs & Long contig & $\mathbf{N}_{\mathbf{5 0}}$ & GC content & Total assembly size \\
BRIP 70780a & $\begin{array}{c}\text { Inverell, New } \\
\text { South Wales }\end{array}$ & 2019 & $22^{\mathrm{a}}$ & $7.02 \mathrm{Mb}$ & $4,257,441 \mathrm{bp}$ & $51.85 \%$ & $52,534,675 \mathrm{bp}$ \\
\hline
\end{tabular}

a One contig (contig number 23) represents the mitochondrial genome.

(Stamatakis 2014), using the concatenated alignment of all four loci based on the GTR substitution model with gamma-distribution rate variation, demonstrated that BRIP 70780a clustered with the ex-epitype strain of M. phaseolina (CBS 205.47) (data not shown). The DNA was also used for constructing an Illumina Nextera DNA Flex library according to manufacturer instructions. The library was sequenced using the Illumina MiSeq platform, using a 600-cycle paired-end V3 reagents kit, which yielded approximately 22,225,240 sequences totaling $4.6 \mathrm{~Gb}$, with mean base quality of 33.1, estimated using FastQC on the Galaxy Australia portal (Afgan et al. 2016). The size of the genome was estimated, using Jellyfish v.2.2.3 (Marçais and Kingsford 2011), to be approximately $53 \mathrm{Mb}$.

High-molecular weight DNA for Nanopore sequencing was obtained by grinding approximately $2 \mathrm{~g}$ of fresh mycelia with a mortar and pestle, followed by a customized cetyltrimethylammonium bromide (CTAB) extraction method (Jones et al. 2019a). Care was taken to avoid DNA shearing while the DNA underwent lysis with CTAB, purification with chloroform, and precipitation with CTAB. Instead of gel purification, the DNA was further purified with chloroform and size-selected for fragments larger than $25 \mathrm{~kb}$, using a Short Read Eliminator Kit (Circulomics) (Jones et al. 2019b). DNA quality was assessed by $1 \%$ agarose gel electrophoresis, was quantified using a Qubit v.3.0 fluorometer and Nanodrop 1000 (both ThermoFisher Scientific). A native DNA sequencing library was constructed with the ligation kit SQK-LSK109 according to manufacturer instructions (Oxford Nanopore Technologies). Sequencing was performed on a FLO-MIN106 R9.4.1 revD flow cell, generating $9.72 \mathrm{~Gb}$ of data with an $\mathrm{N}_{50}$ of $43.50 \mathrm{~kb}$. Fast5 files were base-called to fastq with Guppy 3.1.5 (Oxford Nanopore Technologies) (Wick et al. 2019). Fastq reads were then filtered for quality of Q7 and above.

For de novo assembly, the Maryland Super Read Cabog Assembler (MaSuRCA) v.3.3.3 was used (Zimin et al. 2013). Both the long-read MinION (Oxford Nanopore Technology) and short-read Illumina sequencing datasets were used for assembly, as MaSuRCA is a hybrid assembler. The size of the draft genome assembly was $52.5 \mathrm{Mb}$ with an $\mathrm{N}_{50}$ value of $4,257,441 \mathrm{Mb}$ (Table 1). Benchmarking Universal Single-Copy Orthologs (BUSCO, v.3.0.2) (Simão et al. 2015) was used to assess the completeness of the assembly based on the BUSCOs for Fungi (fungi_odb9) dataset. The genome was estimated to be $99.3 \%$ complete, with 288 complete BUSCOs, one fragmented BUSCO, and one missing BUSCO from a total of 290 BUSCO groups searched. The genome was annotated using BRAKER v.2.1.4 (Hoff et al. 2016), using a protein fasta file from M. phaseolina MS6 (Islam et al. 2012) as a reference, which predicted a total of 14,471 genes. RepeatModeler v.1.0.11 was used to de novo annotate the repetitive elements in the genome. Repeats from RepeatModeler and RepBase library v.20.04 (Bao et al. 2015) were concatenated and were used as input for RepeatMasker v.4.0.9 to soft mask identified repeat elements. It was determined that $19.2 \%$ of the genome was covered in repeats with 779 long interspersed nuclear elements, 4,625 long terminal repeat elements, and 1,909 DNA elements.

The genome resource of $M$. phaseolina reported here is the first from sorghum, reducing the knowledge gap on genus Macrophomina, and will allow for the identification of loci related to the pathogenicity of the fungus and help in identifying genetic variants, which will aid in development of molecular diagnostics tools.

\section{Accession Numbers}

The draft assembly of $M$. phaseolina BRIP 70780a has been deposited in the National Center for Biotechnology Information (NCBI) GenBank database under accession number PRJNA577531. Raw Illumina MiSeq reads and Nanopore reads have been deposited in NCBI SRA with the accession number PRJNA577531. The genome annotation and repeat 
content annotation have been deposited on Zenodo with the doi numbers 10.5281/

zenodo.3581510 and 10.5821/zenodo.3581508.

\section{Author-Recommended Internet Resource}

RepeatMasker and RepeatModeler software: http://www.repeatmasker.org

\section{Acknowledgments}

We thank L. Huth and K. Hadzi for their assistance in Illumina MiSeq work and E.

Adorada and P. Buyoyu for their invaluable help in the lab.

\section{Literature Cited}

Afgan, E., Baker, D., vanden Beek, M., Blankenberg, D., Bouvier, D., Čech, M., Chilton, J., Clements, D., Coraor, N., Eberhard, C., Grüning, B., Guerler, A., Hillman-Jackson, J., Von Kuster, G., Rasche, E., Soranzo, N., Turaga, N., Taylor, J., Nekrutenko, A., and Goecks, J. 2016. The Galaxy platform for accessible, reproducible and collaborative biomedical analyses: 2016 update. Nucleic Acids Res. 44 (W1):W3-W10.

Bandara, Y. M. A. Y., Weerasooriya, D. K., Liu, S., and Little, C. R. 2018. The necrotrophic fungus Macrophomina phaseolina promotes charcoal rot susceptibility in grain sorghum through induced host cell-wall-degrading enzymes. Phytopathology 108:948-956.

Bao, W., Kojima, K. K., and Kohany, O. 2015. Repbase Update, a database of repetitive elements in eukaryotic genomes. Mob. DNA 6:11.

Burkhardt, A. K., Childs, K. L., Wang, J., Ramon, M. L., and Martin, F. N. 2019. Assembly, annotation, and comparison of Macrophomina phaseolina isolates from strawberry and other hosts. BMC Genomics 20:802.

Carbone, I., and Kohn, L. M. 1999. A method for designing primer sets for speciation studies in filamentous ascomycetes. Mycologia 91:553-556.

Crous, P. W., Groenewald, J. Z., Risède, J.-M., Simoneau, P., and Hywel-Jones, N. L. 2004. Calonectria species and their Cylindrocladium anamorphs: Species with sphaeropedunculate vesicles. Stud. Mycol. 50:415-430.

de Hoog, G. S., and Gerrits van den Ende, A. H. G. 1998. Molecular diagnostics of clinical strains of filamentous Basidiomycetes. Mycoses 41:183-189.

Hoff, K. J., Lange, S., Lomsadze, A., Borodovsky, M., and Stanke, M. 2016. Braker1: Unsupervised rna-seq-based genome annotation with genemark-et and augustus. Bioinformatics 32:767-769.

Hossen, Q. M. M., Islam, M. S., Emdad, E. M., Haque, M. S., Alam, M. M., and Alam, M. 2019. Whole-genome optical mapping: Improving assembly of Macrophomina phaseolina MS6 through spanning of twelve blunt end chromosomes by obviating all errors and misassembles. Afr. J. Biotechnol. 18:1031-1043.
Islam, M. S., Haque, M. S., Islam, M. M., Emdad, E. M., Halim, A., Hossen, Q. M., Hossain, M. Z., Ahmed, B., Rahim, S., Rahman, M. S., Alam, M. M., Hou, S., Wan, X., Saito, J. A., and Alam, M. 2012. Tools to kill: Genome of one of the most destructive plant pathogenic fungi Macrophomina phaseolina. BMC Genomics 13:493-509.

Jones, A., Nagar, R., Sharp, A., and Schwessinger, B. 2019a. High-molecular weight DNA extraction from challenging fungi using CTAB and gel purification. Protocols.io dx.doi.org/10.17504/protocols.io.5isg4ee. Published online.

Jones, A., Purushotham, N., Nasim, J., and Schwessinger, B. 2019b. DNA clean-up and size selection for long-read sequencing. Protocols.io dx.doi.org/10.17504/ protocols.io.17506kahcse. Published online.

Marçais, G., and Kingsford, C. 2011. A fast, lock-free approach for efficient parallel counting of occurrences of k-mers. Bioinformatics 27:764-770.

Sarr, M., Ndiaye, M., Groenewald, J., and Crous, P. 2014. Genetic diversity in Macrophomina phaseolina, the causal agent of charcoal rot. Phytopathol. Mediterr. 53:250-268.

Simão, F. A., Waterhouse, R. M., loannidis, P., Kriventseva, E. V., and Zdobnov, E. M. 2015. BUSCO: Assessing genome assembly and annotation completeness with single-copy orthologs. Bioinformatics 31:3210-3212.

Stamatakis, A. 2014. RAxML version 8: A tool for phylogenetic analysis and postanalysis of large phylogenies. Bioinformatics 30:1312-1313.

White, T. J., Bruns, T., Lee, S., and Taylor, J. 1990. Amplification and direct sequencing of fungi ribosomal RNA genes for phylogenetics. Pages 315-322 in: A Guide to Methods and Applications. PCR Protocols Academic, San Diego, CA, U.S.A.

Wick, R. R., Judd, L. M., and Holt, K. E. 2019. Performance of neural network basecalling tools for Oxford Nanopore sequencing. Genome Biol. 20:129.

Woudenberg, J. H. C., Aveskamp, M. M., de Gruyter, J., Spiers, A. G., and Crous, P. W. 2009. Multiple Didymella teleomorphs are linked to the Phoma clematidina morphotype. Persoonia 22:56-62.

Zimin, A. V., Marçais, G., Puiu, D., Roberts, M., Salzberg, S. L., and Yorke, J. A. 2013. The MaSuRCA genome assembler. Bioinformatics 29:2669-2677. 